\title{
How large are dissipative effects in noncritical Liouville string theory?
}

\author{
John Ellis \\ Theoretical Physics Division, CERN, CH-1211 Geneva 23, Switzerland \\ N. E. Mavromatos \\ Theoretical Physics Group, Department of Physics, King's College London, Strand, London WC2R 2LS, United Kingdom \\ D. V. Nanopoulos \\ Department of Physics, Texas A \& M University, College Station, Texas 77843, \\ Astroparticle Physics Group, Houston Advanced Research Center (HARC), Mitchell Campus, Woodlands, Texas 77381, \\ and Chair of Theoretical Physics, Academy of Athens, Division of Natural Sciences, 28 Panepistimiou Avenue, Athens 10679, Greece
}

(Received 1 August 2000; published 29 December 2000)

\begin{abstract}
In the context of non-critical Liouville strings, we clarify why we expect non-quantum-mechanical dissipative effects to be $\mathcal{O}\left(E^{2} / M_{P}\right)$, where $E$ is a typical energy scale of the probe, and $M_{P}$ is the Planck scale. In Liouville strings, energy is conserved at best only as a statistical average, as distinct from Lindblad systems, where it is strictly conserved at an operator level, and the magnitude of dissipative effects could only be much smaller. We also emphasize the importance of nonlinear terms in the evolution equation for the density matrix, which are important for any analysis of complete positivity.
\end{abstract}

DOI: 10.1103/PhysRevD.63.024024

PACS number(s): 04.50.+h, 04.62. $+\mathrm{v}$

\section{INTRODUCTION}

Motivated by speculations concerning quantum gravity, some years ago a modification of conventional quantum mechanics was proposed [1], which admits dissipative phenomena such as transitions from pure to mixed states. This approach postulates the appearance of a non-Hamiltonian term in the quantum Liouville equation describing the evolution of the density matrix:

$$
\dot{\rho}(t)=i[\rho, H]+\delta H \rho .
$$

A derivation of this equation based on a string-inspired treatment of quantum fluctuations in space-time has also been presented [2]. This formalism was applied initially to simple two-state systems such as the neutral kaon system $[1,3,4]$. Arguments have been presented that the magnitude of such dissipative effects could be suppressed minimally by a single power of the gravitational scale $M_{P} \sim 10^{19} \mathrm{GeV}$, i.e.,

$$
\delta H=\mathcal{O}\left(\frac{E^{2}}{M_{P}}\right)
$$

where $E$ is a typical energy scale of the probe system which experiences quantum-gravitational induced decoherence during its propagation through the quantum-gravity "medium" [5]. In particular, the estimate (2) was argued to apply within a non-critical Liouville string model for this foamy spacetime medium. If the estimate (2) is indeed correct, such modifications of quantum mechanics might be accessible to experiment in the foreseeable future, for example in the neutral kaon system, which offers one of the most sensitive microscopic tests of quantum mechanics $[1,3,4]$.

A new arena for testing quantum mechanics has now been opened up by neutrino oscillations, and atmospheric-neutrino data have recently been used [6] to constrain dissipative ef- fects within a Lindblad formalism [7]. However, it has been pointed out [8] that the order-of-magnitude estimate (2) is not applicable to modifications of quantum mechanics described by the Lindblad formalism [7], because the energy scale $E$ appearing in Eq. (2) is no longer the absolute energy of the probe, but rather the energy variance $\Delta E \equiv\left|E_{1}-E_{2}\right|$ of the two-state system:

$$
\delta H=\mathcal{O}\left(\frac{\Delta E^{2}}{M_{P}}\right)
$$

It is therefore crucial $[6,8]$ to know whether the Lindblad formalism [7] applies to the type of of open quantummechanical system provided by a probe propagating through space-time foam.

There are three key requirements for deriving the Lindblad formalism [7], namely energy conservation at the operator level, unitarity and entropy production. However, we have pointed out previously [2] within our stringy approach to quantum gravity that, although unitarity and entropy increase follow from generic properties of the world-sheet renormalization group for the Liouville string, energy conservation should be interpreted at best as a statistical property of expectation values, and even this may be violated in cases with potential physical interest, such as a D-brane model for space-time foam. Thus we do not believe that the Lindblad formalism [7] is directly applicable to propagation through space-time foam, since energy conservation is not imposed at the operator level. It is this difference that permits the magnitude of the dissipative effects to be of order given in Eq. (2), larger than that, Eq. (3), suggested in [8]. We also argue that Eq. (1) contains important nonlinear effects, which are potentially significant [9] for the analysis [10] of complete positivity. 


\section{DISSIPATIVE DYNAMICS IN THE LINDBLAD FORMALISM}

We first give various definitions of the concepts used in the following. We consider a quantum-mechanical system with a density matrix $\rho$, in dissipative interaction with an environment. In the case of a pure state described by a wave vector $|\Psi\rangle$, the von Neumann density matrix operator is given by

$$
\rho=|\Psi\rangle\rangle \Psi \mid
$$

and in a representation $\{a\}$ the matrix elements of $\rho$ are

$$
\rho\left(a, a^{\prime}\right) \equiv\left\langle a^{\prime} \mid \Psi\right\rangle\langle\Psi \mid a\rangle .
$$

In general, for open systems one cannot always define a state vector, in which case the density matrix is defined over an ensemble of theories $\mathcal{M}$ :

$$
\rho \equiv \operatorname{Tr}_{\mathcal{M}}|\Psi\rangle\langle\Psi|
$$

In the coordinate representation, $\{a\}=\{\vec{x}\}$, the diagonal element $\rho(x, x ; t)$ is the probability density $\mathcal{P}(x, t)$, which is given in the pure-state case by the wave function at the time $t$ :

$$
\rho(x, x ; t) \equiv \mathcal{P}(x, t)\left(=|\Psi(x, t)|^{2}\right) \geqslant 0
$$

For well-defined representations $\{a\}$ one must have the positivity property

$$
\rho(a, a) \equiv \rho_{a}>0
$$

Therefore, to define an appropriate density matrix, the operator $\rho_{a}$ must have positive eigenvalues in the state space $\{a\}$ at any time $t$. Even if the matrix $\rho(t=0)$ has positive eigenvalues, it is not guaranteed that the time-evolved matrix $\rho(t)=\omega(t) \rho(0)$ necessarily also has positive eigenvalues. This requirement of simple positivity (SP) has to be imposed, and restricts the general form of the environmental entanglement. Further restrictions apply when one considers $\{a\}$ as coordinates of a subsystem within a larger system interacting with the environment, namely the requirements of complete positivity (CP) [10].

In the particular case of a linear dissipative system with energy conservation, the Lindblad formalism [7] applies; i.e., the quantum evolution is governed by a Markov-type process described by an equation of the form

$$
\begin{aligned}
\dot{\rho}(t)= & i[\rho, H]+\delta H[\rho], \\
\delta H \equiv & 2 \sum_{i} \mathcal{D}_{i}^{(d)} b_{i} \rho(t) b_{i}^{\dagger} \\
& -\sum_{i} \mathcal{D}_{i}^{(d)}\left(b_{i}^{\dagger} b_{i} \rho(t)+\rho b_{i}^{\dagger} b_{i}\right), \\
\mathcal{D}_{i}^{(d)} \geqslant & 0,
\end{aligned}
$$

which maintains SP. Here $H$ denotes the appropriate Hamiltonian operator of the subsystem, the positive coefficients $\mathcal{D}^{(d)}$ are associated to diffusion, and it can be shown that the extra (non-Hamiltonian) terms in the evolution equation (9) induce complete decoherence, as recalled by the index $(d){ }^{1}$

Now suppose one demands that energy be conserved at the Hamiltonian operator level, so that one not only requires the statistical average $\operatorname{Tr}(\rho H)$ to be independent of time, but also the absence of explicit time dependence of the operator $H$ :

$$
\frac{\partial H}{\partial t}=0=\frac{d}{d t} \operatorname{Tr}(\rho H) .
$$

This requirement, together with the monotonic increase of the von Neumann entropy $S=-\operatorname{Tr} \rho \ln \rho$, implies, for the environmental operators $b_{i}, b_{i}^{\dagger}$,

$$
\left[b_{i}, H\right]=0, \quad b_{i}=b_{i}^{\dagger} \text {. }
$$

Then, the environmental part $\delta H[\rho]$ of the evolution (9) assumes a double-commutator form $[13,8]$

$$
\delta H=\sum_{i} \mathcal{D}_{i}^{(d)}\left[b_{i},\left[b_{i}, \rho\right]\right]
$$

Clearly, for a two-state system, with energy levels $E_{n}, n$ $=1,2$, the only non-trivial Lindblad operators satisfying Eq. (11) are of the form $b_{i} \propto H$.

This simplified case with only one operator suffices for our purposes [8], and we restrict our discussion to this case. One may then estimate the magnitude of the dissipative effects by considering a statistical average of $\delta H$ with respect to a complete basis of states, which can be taken as the energy eigenstates $\{|m\rangle\}$ of the Hamiltonian $H, H|m\rangle$ $=E_{m}|m\rangle, m=1,2$. In such a case one has [8]

$$
\begin{aligned}
\langle\langle\delta H\rangle\rangle & =2 \sum_{m, n=1}^{2}\langle m|\rho| n\rangle\langle n|\rho| m\rangle E_{n}\left(E_{n}-E_{m}\right) \\
& =2\langle 1|\rho| 2\rangle\langle 2|\rho| 1\rangle\left(E_{2}-E_{1}\right)^{2}
\end{aligned}
$$

and thus one gets the order of magnitude (3) for the possible dissipative effects [8]. A similar conclusion is reached if one uses position eigenstates $|q\rangle$ as a basis, which is the case of spontaneous localization models $[12,13,8]$, instead of energy eigenstates. In this case, the dissipative effects are proportional to the position variance $\Delta q$, i.e., the separation $\left|q_{1}-q_{2}\right|$ between the centers of the wave packets. ${ }^{2}$ These

\footnotetext{
${ }^{1}$ It should be noticed that the density-matrix evolution equation (9) can be recast, using Eq. (6), as a state-vector evolution equation of stochastic Ito type [12], if one wishes. We prefer the more general density-matrix formalism, because the concept of a state vector is not always well defined, particularly in our quantum-gravity foam context $[1,2]$.

${ }^{2}$ For instance, of the corresponding neutrino probes in the example discussed in [6].
} 
estimates are considerably smaller than our estimate (2), so it is important to address carefully the question how the Liouville formalism differs from the generic Lindblad formalism.

\section{DISSIPATIVE DYNAMICS IN NON-CRITICAL STRINGS}

For the benefit of the non-expert reader, we now review how a dissipative evolution equation of the form (1) arises in the context of non-critical string, and how energy is not conserved at an operator level, but at best as a statistical average. We also discuss the special circumstances under which Eq. (9) may be obtained, in the hope of clarifying the essential differences between the two approaches.

\section{A. Non-critical strings in flat world sheets}

Critical string theory is described by a conformal field theory $S^{*}$ on the two-dimensional world sheet $\Sigma$. We describe non-critical string in terms of a generic non-conformal field theory ( $\sigma$ model) on $\Sigma$. This is perturbed away from conformal symmetry (criticality) by deformations that are slightly relevant (in a world-sheet renormalization-group sense), with vertex operators $\left\{V_{i}\right\}$ and couplings $\left\{g_{i}\right\}$, which parametrize the space of possible theories:

$$
S=S^{*}+\int_{\Sigma} d^{2} \sigma g^{i} V_{i}
$$

The renormalization-group $\beta$ function for a coupling $g^{i}$ on a flat world sheet reads

$$
\hat{\beta}^{i}=-\varepsilon g^{i}+\beta^{i}, \quad \beta^{i}=C_{j k}^{i} g^{j} g^{k}+\cdots
$$

where $\varepsilon \rightarrow 0^{+}$is a regularizing parameter; e.g., in dimensional regularization, the dimensionality of the world sheet is assumed to be $d=2-\varepsilon$. As is clear from Eqs. (15), $\varepsilon$ plays the role of a small anomalous dimension that makes the operator $V_{i}$ slightly relevant. According to standard renormalization theory, counterterms can be expanded in poles in $\varepsilon$, and we make the standard dimensional-regularization assumption that only single poles matter, while higher-order poles cancel among themselves.

The fact that the world sheet $\Sigma$ is in general curved implies that one has to choose a regularization scale $\mu$ which is local on the world sheet [2], as is standard in stringy $\sigma$ models [14]. The crucial next step in our approach [2] is to promote the scale $\mu(\sigma)$ to a dynamical world-sheet field $\phi$ which should appear in a world-sheet path integral, taking the form of Liouville string theory [15]. Because of its target-space signature, which is negative for the supercritical strings [16] we consider, the world-sheet zero mode of this field is identified with target time.

The conformal invariance of the stringy $\sigma$ model may be restored by Liouville dressing [15], leading to the following equation for the dressed couplings $\lambda^{i}\left(\phi, g^{i}\right)$ :

$$
\ddot{\lambda}^{i}+Q \quad \dot{\lambda}^{i}=-\hat{\beta}^{i}
$$

where $Q^{2}$ is the central-charge deficit of the non-conformal theory $[15,16]{ }^{3}$ When one identifies the Liouville field equation (16) with a curved-world-sheet renormalization-group flow [2], the Liouville-dressed couplings $\lambda^{i}$ may be identified with appropriately renormalized $\sigma$-model couplings $g_{i}$ on curved world sheets.

As was shown in [2], the summation over possible topologies of the world sheet induces canonical quantization of the theory-space coordinates $\left\{g^{i}\right\}$, much as the couplings in local field theories are quantized in the presence of wormhole fluctuations in space-time [17]. This canonical quantization follows from a certain set of Helmholtz conditions in theory space [2], which are obeyed provided the $\beta^{i}$ functions obey the gradient-flow property

$$
\hat{\beta}^{i}=G^{i j} \frac{\partial C[g]}{\partial g^{j}}
$$

where $C[g, t]$ is the Zamolodchikov $C$ function [18], a renormalization-group invariant combination of averages [with respect to Eq. (14)] of components of the world-sheet stress tensor of the deformed $\sigma$ model, $G^{i j}$ is the inverse of the Zamolodchikov metric in theory space,

$$
G_{i j}=\left\langle V_{i} V_{j}\right\rangle,
$$

and the angular brackets denote any expectation value over the partition function of the deformed $\sigma$ model (14), summed over world-sheet genera. The Zamolodchikov $C$ function plays the role of the off-shell effective target-space action in string theory, which allows the identification

$$
C[g]=\int d t\left(p_{i} \dot{g}^{i}-E\right)
$$

where $E$ is the Hamiltonian operator of the string matter. In critical string theories, the couplings $g^{i}$ are exactly marginal, $\beta^{i}=0$, and this formalism has trivial content, but this is no longer the case [2] when one goes beyond critical strings.

Renormalizability of the world-sheet $\sigma$ model implies the scale independence of physical quantities, such as the partition function or the density matrix $\rho\left(g_{i}, p^{j}, t\right)$ of a string moving in the background parametrized by the $\left\{g^{i}\right\}$, viewed as generalized coordinates in string theory space, with the $p_{j}$ the canonically conjugate momenta in this space, which are associated with the vertex operators $V_{j}$ in a subtle sense [2]. Let $t=\ln \mu_{0}$ be the (zero mode of) the world-sheet renormalization-group scale. Since the elements of the density matrix are physical quantities, and hence independent of the world-sheet scale, they must obey [2] the following renormalization-group equation:

$$
\frac{d}{d t} \rho\left(g_{i}, p^{j}, t\right)=\frac{\partial}{\partial t} \rho+\dot{g}^{i} \frac{\partial}{\partial g^{i}} \rho+\dot{p}_{i} \frac{\partial}{\partial p_{i}} \rho=0
$$

\footnotetext{
${ }^{3}$ We used this approach in [2] to derive a stochastic FokkerPlanck equation with diffusion for the corresponding probability distribution in the theory space $\left\{\lambda^{i}\right\}$.
} 
where an overdot denotes a partial derivative with respect to $t$. The total $t$ derivative above incorporates $t$ dependence both explicitly and implicitly, through running couplings and generalized momenta. ${ }^{4}$

As an example how non-critical string dynamics enters, one may consider the reduced density matrix $\rho_{s}\left(g_{s}^{i}, p_{s, j}, t\right)$ of an effective string theory describing lowest-level string modes characterized by a subset $\left\{g_{s}^{i}\right\} \in\left\{g^{i}\right\}$ of couplings to operators $\left\{V_{s}^{i}\right\}$ that are not exactly marginal. These deformations are remnants of mixtures of these lowest-level modes with higher (Planckian) modes of the string, which are initially exactly marginal. However, this property is lost when the higher-level modes are integrated over, so that the remnant $\left\{V_{s}^{i}\right\}$ provide a non-trivial background "environment" for the observable lowest-level modes to propagate through. This "gravitational environment" of higher-level states is quantized when one sums over world-sheet genera. We have made case studies of such systems, based on stringy black holes in two space-time dimensions [19], ${ }^{5}$ and higherdimensional analogues provided by the recoil [11] of D-branes [20] when struck by a light propagating string state. At present, we only discuss some generic properties of this Liouville approach to space-time foam [2].

The non-critical subsystem $\left\{g_{s}\right\}$ acquires non-trivial dynamics through Eq. (20), since the $\beta^{i}$ functions are nontrivial. Summing over genera, taking into account the canonical quantization of the theory space mentioned above, and identifying the Liouville renormalization-group scale as the target time variable [2], one arrives at the following evolution equation for the reduced density matrix operator $\rho_{s}$ of the observable, propagating, localized, lowest-level modes of the effective string theory:

$$
\partial_{t} \rho_{s}\left(g_{s}, p_{s}, t\right)=i\left[\rho_{s}, \mathcal{H}\right]+i \hat{\beta}_{s}^{i} G_{i j}\left[g_{s}^{j}, \rho_{s}\right]
$$

where $\mathcal{H}$ is a Hamiltonian operator for the subsystem consisting of propagating string modes, and the second term on the right-hand side of Eq. (21) is an explicit string representation for $\delta H$ in Eq. (1). It is clear that our fundamental equation (21) stems from the requirement of renormalizability (20).

This description has several important properties, which we now describe. For notational convenience in what follows, we omit the sub-index $s$ in the couplings and conjugate momenta, i.e., $g_{s} \rightarrow g_{i}$, etc., but we always imply quantities in the subsystem $s$, unless otherwise stated. We remind the

\footnotetext{
${ }^{4}$ Here we study the general consequences of Eq. (20), although it is possible that only the diagonal elements of the density matrix in the coordinate representation, which may be interpreted as probability densities, are in fact measurable physical quantities, in which case the requirement (20) should be applied only to the world-sheet partition function of the stringy $\sigma$ model.

${ }^{5}$ In this case the environment is provided by discrete delocalized solitonic states, which mix explicitly with lowest-level propagating matter states of the two-dimensional string in marginal deformations of the two-dimensional black hole, as a reflection of infinitedimensional $W_{\infty}$ gauge symmetries [2].
}

reader that in the non-critical string theory model discussed here the full system is conformal, and as such the time (renormalization-group) evolution (20) acquires trivial content.

Probability conservation. The total probability $P$ $=\int d p_{l} d g^{l} \operatorname{Tr}\left[\rho\left(g^{i}, p_{j}\right)\right]$ is conserved, because

$$
\dot{P}=\int d p_{l} d g^{l} \operatorname{Tr}\left[\frac{\partial}{\partial p_{i}}\left(G_{i j} \beta^{j} \rho\right)\right]
$$

can receive contributions only from the boundary of phase space, which must vanish for an isolated system.

Entropy growth. The entropy $S=-\operatorname{Tr}(\rho \ln \rho)$ is not conserved, to the extent that relevant couplings with $\beta^{i} \neq 0$ are present,

$$
\dot{S}=\left(\beta^{i} G_{i j} \beta^{j}\right) S,
$$

implying a monotonic increase for unitary world-sheet theories for which $G_{i j}$ is positive definite. We see from Eq. (23) that any running of any coupling will lead to an increase in entropy, and we have interpreted [2] this behavior in terms of quantum models of friction. The increase (23) in the entropy corresponds to a loss of quantum coherence, which is also known in models.

Statistical conservation of energy. The most important property for our purposes here is that energy is at best conserved statistically on the average [2]. In other words, one has explicit time dependence (dissipation) in the Hamiltonian operator of the subsystem, thereby allowing flow of energy to the environment at an operator level. Then, it is at best only the right-hand equality in Eq. (10) that is valid, depending on the specific model. ${ }^{6}$

To see this, we recall, as discussed above Eq. (20), that the renormalizability of the $\sigma$ model implies $d / d t \operatorname{Tr} \rho=0$. Since we identify the target time with the renormalizationgroup scale $t$ on the world sheet [2], $\partial_{t} \operatorname{Tr} \rho_{s}$ may be expressed in terms of the renormalized couplings $g^{i}$ by means of the evolution equation (21). We now compute

$$
\frac{\partial}{\partial t}\langle\langle E\rangle\rangle=\frac{\partial}{\partial t} \operatorname{Tr}(E \rho)=\left\langle\left\langle\partial_{t}\left(E-\beta^{i} G_{i j} \beta^{j}\right)\right\rangle\right\rangle
$$

where $E$ is the Hamiltonian operator, and $\langle\langle\ldots\rangle\rangle$ $\equiv \operatorname{Tr}[\rho(\cdots)]$. In deriving this result, we took into account the evolution equation (21) and the quantization rules in theory space [2],

$$
\left[g^{i}, g^{j}\right]=0, \quad\left[g^{i}, p^{j}\right]=-i \delta^{i j},
$$

as well as the fact that in string $\sigma$ models the quantum operators $\beta^{i} G_{i j}$ are functionals only of the coordinates $g^{i}$, and not of the generalized momenta $p^{i}$. We also note that, in our approach, the total time derivative of an operator $\hat{Q}$ is given as usual by

\footnotetext{
${ }^{6}$ See the next section for a question mark that hangs over even this statistical equality.
} 


$$
\frac{d}{d t} \hat{Q}=-i[\hat{Q}, E]+\frac{\partial \hat{Q}}{\partial t} .
$$

We recall that total time derivatives incorporate both explicit and implicit renormalization-scale dependence (via running couplings), while partial time derivatives incorporate only the explicit dependence.

Using the $C$-theorem results (17),(19) $[18,2]$ and the formalism developed in [2], it is straightforward to arrive at

$$
\frac{\partial}{\partial t}\langle\langle E\rangle\rangle=\frac{\partial}{\partial t}\left(p_{i} \beta^{i}\right) .
$$

In conventional stringy $\sigma$ models, as a result of world-sheet renormalizability with respect to a "flat" world-sheet cutoff, any dependence on the renormalization group scale in the $\beta^{i}$ functions is implicit through the renormalized couplings, and hence $\partial_{t} \beta^{i}=0$. Moreover, the quantity $p_{i}$ appearing in Eq. (27) may be written in the form

$$
p_{i}=G_{i j} \beta^{j} \sim \sum_{n} C_{i i_{1} \cdots i_{n}} g^{i_{1}} \cdots g^{i_{n}}
$$

where the $C_{i j \ldots}$ are the (totally symmetric) correlators of vertex operators $\left\langle V_{i} V_{j} \ldots\right\rangle$. In the usual case, as a result of the renormalizability of the $\sigma$-model theory, there is no explicit dependence on the world-sheet scale $t$ in such correlators or on $\beta^{i}$, and hence the right-hand side of Eq. (27) vanishes, implying energy conservation on the average.

In this derivation, renormalizability replaces the timetranslation invariance of conventional target-space field theory.

\section{B. Curved world-sheet renormalization and generic Liouville correlators}

An additional feature appears in certain non-critical string theories that involve solitonic structures in their backgrounds, such as $D$ particles [21,11,22]. There are deformations in the set of $\left\{V_{i}\right\}$ that obey a logarithmic conformal algebra [23], rather than an ordinary conformal algebra. As discussed in detail in [24], the field correlators $C_{i_{1} \cdots i_{m}}$ in such logarithmic conformal field theories exhibit explicit dependences on the world-sheet renormalization (time) scale $t$. This, in fact, is essential in guaranteeing the gradient-flow property (17) of the corresponding $\beta$ functions [24], which is crucial for canonical quantization in theory space [2], as we discussed previously. As a result, our previous argument for energy conservation on the average breaks down in theories with logarithmic deformations, because the right-hand side of Eq. (27) is no longer non-zero. Physically, this is explained by the flow of energy from the propagating subsystem to the recoiling $D$-particle background [22].

In standard (critical) string theory, the correlators $C_{i_{1} \cdots i_{m}}$ are associated with elements in the $S$ matrix for particle scattering, and as such should not exhibit any explicit dependence on the time coordinate. In view of their explicit dependence on the world-sheet scale in logarithmic conformal field theories, which in our approach [2] is identified with the target time, such correlators cannot be interpreted as conventional scattering amplitudes in target space, but rather as non-factorizable $\$$ matrix elements. The time dependence also means that, while the initial formulations of quantumgravitational dissipation $[1,3,4]$ assumed energy conservation, this can no longer be guaranteed in string soliton models of space-time foam [11].

There is one more formal reason for relaxing strict energy conservation in Liouville strings, which we review below [25]. The discussion of the previous subsection pertained to flat world sheets, but the situation is different when one considers generic correlators in Liouville strings, because the world sheet has curvature expressed essentially by the dynamical Liouville mode. A more correct approach is to consider renormalization in curved space [14], which leads to new types of counterterms. It is just this feature that may lead to violations of the energy conservation, as happens explicitly in the D-brane case [22,24], which is a particular case of Liouville strings. Here we briefly review the situation, concentrating on those aspects of the formalism relevant to energy conservation, referring the reader interested in more details to the literature $[25,2]$.

We consider the $N$-point correlation function of vertex operators in a generic Liouville theory, viewing the Liouville field as a local renormalization-group scale on the world sheet [2]. Standard computations [26] yield the following form for an $\mathrm{N}$-point correlation function of vertex operators integrated over the world sheet: $V_{i} \equiv \int d^{2} z V_{i}(z, \bar{z})$ :

$$
\begin{aligned}
A_{N} & \equiv\left\langle V_{i_{1}} \cdots V_{i_{N}}\right\rangle_{\mu} \\
& =\Gamma(-s) \mu^{s}\left\langle\left(\int d^{2} z \sqrt{\hat{\gamma}} e^{\alpha \phi}\right)^{s} \widetilde{V}_{i_{1}} \cdots \widetilde{V}_{i_{N}}\right\rangle_{\mu=0}
\end{aligned}
$$

where the tilde denotes removal of the zero mode of the Liouville field $\phi$. The world-sheet scale $\mu$ is associated with cosmological-constant terms on the world sheet, which are characteristic of the Liouville theory, and the quantity $s$ is the sum of the Liouville anomalous dimensions of the operators $V_{i}$ :

$$
s=-\sum_{i=1}^{N} \frac{\alpha_{i}}{\alpha}-\frac{Q}{\alpha}, \quad \alpha=-\frac{Q}{2}+\frac{1}{2} \sqrt{Q^{2}+8} .
$$

The $\Gamma$ function appearing in Eq. (29) can be regularized $[27,2]$ for negative-integer values of its argument by analytic continuation to the complex plane using the the Saaschultz contour of Fig. 1.

To see technically why the above formalism leads to a breakdown in the interpretation of the correlator $A_{N}$ as a string amplitude or $S$-matrix element, which in turn leads to the interpretation of the world-sheet partition function as a probability density rather than a wave function in target space, one first expands the Liouville field in (normalized) eigenfunctions $\left\{\phi_{n}\right\}$ of the Laplacian $\Delta$ on the world sheet

$$
\phi(z, \bar{z})=\sum_{n} c_{n} \phi_{n}=c_{0} \phi_{0}+\sum_{n \neq 0} c_{n} \phi_{n}, \quad \phi_{0} \propto A^{-1 / 2},
$$




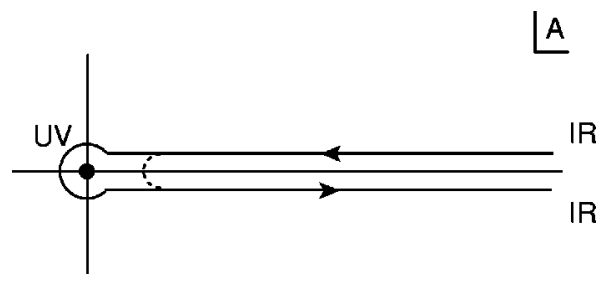

FIG. 1. The contour of integration for the analytically continued (regularized) version of $\Gamma(-s)$ for $s \in Z^{+}$. The quantity $A$ denotes the (complex) world-sheet area. This is known in the literature as the Saalschutz contour, and has been used in conventional quantum field theory to relate dimensional regularization to the BogoliubovParasiuk-Hepp-Zimmermann renormalization method. Upon the interpetation of the Liouville field with target time, this curve resembles closed-time paths in non-equilibrium field theories [28].

where $A$ is the world-sheet area, and

$$
\begin{gathered}
\Delta \phi_{n}=-\epsilon_{n} \phi_{n}, \quad n=0,1,2, \ldots, \\
\epsilon_{0}=0, \quad\left(\phi_{n}, \phi_{m}\right)=\delta_{n m} .
\end{gathered}
$$

The correlation functions (without the Liouville zero mode) appearing on the right-hand side of Eq. (29) take the form [25]

$$
\begin{aligned}
\widetilde{A}_{N} \propto & \prod_{n \neq 0} d c_{n} \exp \left(-\frac{1}{8 \pi} \sum_{n \neq 0} \epsilon_{n} c_{n}^{2}-\frac{Q}{8 \pi} \sum_{n \neq 0} R_{n} c_{n}\right. \\
+ & \left.\sum_{n \neq 0} \alpha_{i} \phi_{n}\left(z_{i}\right) c_{n}\right)\left[\int d^{2} \xi \sqrt{\hat{\gamma}} \exp \left(\alpha \sum_{n \neq 0} \phi_{n} c_{n}\right)\right]^{s}
\end{aligned}
$$

where $R_{n}=\int d^{2} \xi R^{(2)}(\xi) \phi_{n}$. We can compute Eq. (33) if we analytically continue [26] $s$ to a positive integer $s \rightarrow n$ $\in \mathbf{Z}^{+}$. Denoting

$$
f(x, y) \equiv \sum_{n, m \neq 0} \frac{\phi_{n}(x) \phi_{m}(y)}{\epsilon_{n}}
$$

where we use the compact notation $x \equiv\left(x_{1}, x_{2}\right)$ for the world-sheet coordinates, we observe that, as a result of the lack of the zero mode:

$$
\Delta f(x, y)=-4 \pi \delta^{(2)}(x, y)-\frac{1}{A} .
$$

We may choose the gauge condition $\int d^{2} \xi \sqrt{\hat{\gamma}} \widetilde{\phi}=0$, which determines the conformal properties of the function $f$ as well as its "renormalized" local limit

$$
f_{R}(x, x)=\lim _{x \rightarrow y}\left[f(x, y)+\ln d^{2}(x, y)\right]
$$

where $d^{2}(x, y)$ is the geodesic distance on the world sheet. Integrating over $c_{n}$, one obtains

$$
\begin{aligned}
\widetilde{A}_{n+N} \propto \exp \left[\frac{1}{2} \sum_{i, j} \alpha_{i} \alpha_{j} f\left(z_{i}, z_{j}\right)\right. \\
+\frac{Q^{2}}{128 \pi^{2}} \iint R(x) R(y) f(x, y) \\
\left.-\sum_{i} \frac{Q}{8 \pi} \alpha_{i} \int \sqrt{\hat{\gamma}} R(x) f\left(x, z_{i}\right)\right] .
\end{aligned}
$$

We now consider infinitesimal Weyl shifts of the worldsheet metric: $\gamma(x) \rightarrow \gamma(x)[1-\sigma(x)]$. Under these Weyl shifts, the correlator $A_{N}$ transforms as follows $[25,2]$ :

$$
\begin{aligned}
\delta \widetilde{A}_{N} \propto[ & \sum_{i} h_{i} \sigma\left(z_{i}\right)+\frac{Q^{2}}{16 \pi} \int d^{2} x \sqrt{\hat{\gamma}} \hat{R} \sigma(x) \\
& +\frac{1}{\hat{A}}\left\{Q s \int d^{2} x \sqrt{\hat{\gamma}} \sigma(x)+(s)^{2} \int d^{2} x \sqrt{\hat{\gamma}} \sigma(x) \hat{f}_{R}(x, x)\right. \\
& +Q s \iint d^{2} x d^{2} y \sqrt{\hat{\gamma}} R(x) \sigma(y) \hat{\mathcal{G}}(x, y) \\
& -s \sum_{i} \alpha_{i} \int d^{2} x \sqrt{\hat{\gamma}} \sigma(x) \hat{\mathcal{G}}\left(x, z_{i}\right) \\
& -\frac{1}{2} s \sum_{i} \alpha_{i} \hat{f}_{R}\left(z_{i}, z_{i}\right) \int d^{2} x \sqrt{\hat{\gamma}} \sigma(x) \\
& \left.\left.-\frac{Q s}{16 \pi} \iint d^{2} x d^{2} y \sqrt{\hat{\gamma}(x) \hat{\gamma}(y)} \hat{R}(x) \hat{f}_{R}(x, x) \sigma(y)\right\}\right] \widetilde{A}_{N}
\end{aligned}
$$

where the caret denotes transformed quantities, and the function $\mathcal{G}(\mathrm{x}, \mathrm{y})$ is defined as

$$
\mathcal{G}(z, \omega) \equiv f(z, \omega)-\frac{1}{2}\left[f_{R}(z, z)+f_{R}(\omega, \omega)\right]
$$

and transforms simply under Weyl shifts $[25,2]$. We observe from Eq. (38) that if the sum of the anomalous dimensions $s \neq 0$, the "off-shell" effect of non-critical strings, then there are non-covariant terms in Eq. (38), inversely proportional to the finite-size world-sheet area $A$. Thus the generic correlation function $A_{N}$ does not have a well-defined finite limit as $A \rightarrow 0$.

In our approach to string time, we identify [2] the target time as $t=\phi_{0}=-\log A$, where $\phi_{0}$ is the world-sheet zero mode of the Liouville field. The normalization is specified by requiring the canonical form of the kinetic term for $\phi$ in the Liouville $\sigma$ model $[16,2]$. The opposite flow of the target time, as compared to that of the Liouville mode, follows from the "bounce" picture [27,2] for the Liouville flow of Fig. 1. The induced time (world-sheet scale $A$ ) dependences of the correlation functions $A_{N}$ imply the breakdown of their interpretations as well-defined $S$-matrix elements, whenever there is a departure from criticality: $s \neq 0$. 
We now obtain a non-zero contribution to Eq. (27) from an apparent non-trivial explicit $t$ dependence in $\partial_{g^{i}} \beta^{i}$ through the coefficients $C_{i_{1} \cdots i_{m}}^{i}$ of the $\beta^{i}$ functions, associated with the breakdown of their identification as well-defined $S$-matrix elements, in contrast to the conventional string case. As discussed in [2], one can explicitly verify this picture in the case of $D$-particle recoil. The scattering of a lowenergy (string) matter particle off a $D$ particle conserves energy only in the complete system, when the recoil of the $D$ particle is taken properly into account [22]. The recoil degrees of freedom are entangled with the subsystem of lowenergy string matter, and their neglect leads to an explicit violation of the energy conservation condition at an operator level. Energy conservation can at best be imposed in a statistical average sense.

\section{Can one restore the Lindblad formalism in non-critical string?}

We have seen that the non-critical-string time evolution (21) is nothing other than a world-sheet renormalizationgroup evolution equation in coupling constant space $\left\{g^{i}\right\}$ for the $\sigma$ model and, in view of the failure of energy conservation, discussed above, cannot in general be written in the Lindblad form (9). There are, however, some special cases in which the equation can indeed be put in a Lindblad form, but even in such cases, as we shall explain below, the order of magnitude of the dissipative effects is still given by Eq. (2) and not Eq. (3).

We first recall from our above discussion that the noncritical string evolution equation (21) represents diffusive evolution in theory space of the non-critical string $\left\{g^{i}\right\}$. Hence, as explained in detail in [2], there is spontaneous localization in such a space $[12,13]$. Thus, even if the situation resembled that of Lindblad, it would not have been associated with energy-driven diffusion, as was the case discussed in [6], but with spontaneous localization models [8]. This can be seen straightforwardly from the form (21) for the time evolution of the density matrix in non-critical string theory. In this case, there is no "environment operator" that commutes with the Hamiltonian, for the simple reason that the role of $b_{i}$ is played in Liouville strings by various partitions (within a generalized definition of the quantumordering prescription for the operators $g^{i}$ ) of $G_{i j} \beta^{j}$ $=C_{i i_{1} \cdots i_{m}} g^{i_{1}} \cdots g^{i_{m}}$. The operators $g^{i}$ play the role of position operators in a generalized coupling-constant space, and, as such, the $g^{i}$ do not commute with $H$ in general, which depends on the generalized momenta in theory space $p_{i}$. Hence our non-critical string model for decoherence does not respect the Lindblad criterion (11).

It can easily be seen that a double-commutator structure could only arise in a situation where only the linear anomalous-dimension terms are kept in $\beta^{i}=y^{i} g^{i}+\cdots$, with no sum over $i$. In that case, making the antisymmetric ordering prescription denoted by : $\cdots$, the diffusive term in the Liouville string evolution equation (21) does acquire a double-commutator structure

$$
: \delta H: \sim y^{i}\left[g^{i},\left[g^{i}, \rho\right]\right] .
$$

In the two-state case studied in $[6,8]$, this leads to an estimate of the dissipative effects as being of order $\mathcal{O}\left(y^{i}\left(\Delta g^{i}\right)^{2}\right)$, where $\Delta g^{i}$ is the variance in theory space, which should be distinguished from real position variances. Indeed, in real space, $\Delta g^{i}$ may be considered of order 1 , and this leaves the anomalous dimension factor $y^{i}$ to determine the order of the effect. This is due to the fact that, as discussed in [2], the Liouville string case is characterized by the appearance of pointer states [13] in theory space, namely wave packets with $\Delta g^{i} \sim h_{s}$, where $h_{s}$ is the "Planck constant" in theory space, which is found [22] to be proportional to the string coupling $g_{s}$. This latter property can easily be understood from the fact that quantization in theory space is induced in our approach by higher-genus topologies on the world-sheet and, hence, string-loop interactions [2]. In conventional string models, $g_{s}$ is of order 1 , namely $g_{s}^{2} / 4 \pi=1 / 20$. However, one may consider more general cases in which $g_{s}$ is a phenomenological parameter, to be constrained by data, e.g., those on neutrinos [6].

In generic non-critical string models, the operators corresponding to the $g^{i}$ are $(1,1)$ on the world sheet, so that $y^{i}$ $=0$, but not exactly marginal, which implies that only the three- and higher-point-function contributions are non-zero in $\beta^{i}=C_{j k}^{i} g^{j} g^{k}+\cdots$. The latter terms clearly do not lead to a double commutator structure $(40,12)$. The order of the corresponding effects can, however, be estimated by the fact that the correlators $C_{i_{1} \cdots i_{m}}^{i}$ are viewed as (non-factorizable) $\$$-matrix elements in target space, and as such can be expanded in a power series in $\alpha^{\prime} k^{2}$ in the interesting closedstring case, where $k$ is a typical target-space four-momentum scale, $\alpha^{\prime}=1 / M_{s}^{2}$ the Regge slope, and $M_{s}$ the string scale. This yields once more the estimate (2) for string-induced dissipative effects.

This estimate is supported by many specific examples. For instance, in the context of the two-dimensional blackhole model of [19], the analysis of [2] showed that the $e x$ actly marginal world-sheet correlators involve necessarily the coupling of low-energy propgating modes with Planckian solitonic modes. The world-sheet correlators involving the latter are suppressed to leading order by a single power of the Planck mass $M_{P} .{ }^{7}$ This coupling is necessitated by stringy gauge symmetries, specifically $W_{\infty}$ symmetries, which were argued in [2] to be responsible for maintaining quantum coherence at the microscopic string level, but not at the level of the low-energy effective theory relevant to observation. This in turn implies that the splitting between lowenergy propagating modes and quantum-gravitational modes is suppressed by a single inverse power of $M_{s}$ or $M_{P}$, leading again to the above estimate for the magnitude of $C_{i_{1} \cdots i_{n}}^{i}$, where the $g^{i_{n}}$ refer to low-energy propagating matter modes. In a similar spirit, the recoil approach to D-brane and string scattering [21,11], which is another example of a gravita-

\footnotetext{
${ }^{7}$ Strictly speaking, the string scale $M_{s}$, but we do not draw the distinction here.
} 
tional medium, also suggests that dissipative effects are suppressed by a single inverse power of the gravitational mass scale, as in Eq. (2).

It goes without saying, however, that there is always the possibility of a cancellation in some specific case, so that the suppression is by some higher power of the gravitational scale as in Eq. (3), but we see no reason why this should be generic.

\section{NONLINEARITIES AND COMPLETE POSITIVITY}

We conclude with some remarks on nonlinearities in Liouville string dynamics. It has been pointed out [10] that the naive extension of a single-particle approach to twoparticle systems may not respect $\mathrm{CP}$, and constraints on the parametrization of dissipative effects in single-particle systems have been proposed. However, as we argue below, it may be an oversimplification to ignore the likelhiood of nonlinearities in the quantum-gravitational framework, which would require the issue of complete positivity [10] to be re-evaluated [9].

An important indication of the possible importance of nonlinear environmental effects comes from the form of the evolution equation (21) for the reduced density matrix in the context of Liouville strings. The appearance of the $G_{i j}$ $\sim\left\langle V_{i} V_{j}\right\rangle$ term given by Eq. (18) in the dissipative part of Eq. (21) is a signature of nonlinear Hartree-Fock evolution, since the expectation values $\langle\cdots\rangle$ are to be evaluated with respect to a world-sheet partition function $\Psi\left[g^{i}, t\right]$ of the string that is resummed over genera. According to the detailed discussion in [29], such a resummed world-sheet partition function may be identified as the trace of the density matrix $\rho$, with a probability distribution in theory space. As such, the dissipative aspects of the evolution exhibit a nonlinear integrodifferential form

$$
\partial_{t} \rho_{s} \ni\left(\operatorname{Tr}_{g_{i}} \rho_{s} V_{i} V_{j}\right) \hat{\beta}^{i}\left[g_{s}^{j}, \rho_{s}\right]
$$

where $\operatorname{Tr}_{g_{i}}$ denotes a partial trace over quantum fluctuations about the string background $\left\{g_{i}\right\}[2,22]$,

$$
\begin{aligned}
\operatorname{Tr}_{g_{i}}(\cdots) \equiv & \int \sqrt{\Gamma^{-1}} d \alpha_{i} \exp \left(-\frac{\alpha_{i} G^{i j} \alpha_{j}}{\Gamma}\right) \\
& \times \exp \left(-S^{*}+\int_{\Sigma}\left(g_{i}+\alpha_{i}\right) V_{i}\right)(\cdots),
\end{aligned}
$$

and the quantum fluctuations $\alpha_{i}$ are of stochastic type, with a Gaussian probability distribution in theory space, as a result of the sum over world-sheet topologies.

Equation (41) should be understood as an operator equation in theory space. Its form is consistent with the fact that, in the $\left\{g_{i}\right\}$ representation, $G_{i j} \sim\left(\delta^{2} / \delta g_{i} \delta g_{j}\right) \operatorname{Tr}_{g_{i}} \rho_{s}$, as follows from canonical quantization in theory space [2,22], according to which $V_{i} \rightarrow-i \delta / \delta g^{i}$. Near a fixed point in theory space, as is reached at asymptotically large times $t \rightarrow \infty$, where perturbation theory in $\left\{g_{s}\right\}$ is valid, it might be a sufficiently good approximation to ignore the nonlinearities and parametrize the terms $G_{i j} \beta^{j}$ by some "constants." However, one should not expect this linearization to be valid throughout the evolution, e.g., at early times after the production in $\phi$ decay of a correlated $K^{0}-\bar{K}^{0}$ system.

We do not pursue this point here, but emphasize that, in our view, such nonlinearities need to be understood [9] before using complete positivity to impose restrictions on the parametrization of $\delta H$ for single-particle systems that go beyond those imposed by simple positivity [10]. They may also be important for the understanding of the energy-momentum conservation issue raised in [4].

\section{OUTLOOK ON THE NEUTRINO AND OTHER EXPERIMENTS}

We have reviewed in this paper why we expect that dissipative effects on isolated systems due to quantumgravitational effects might be as large as $\mathcal{O}\left(E^{2} / M_{P}\right)$. This estimate is potentially very encouraging for several classes of experiments, possibly including neutrinos.

As has been discussed extensively, present and nearfuture kaon experiments may be sensitive to an effect of this magnitude $[1,3,4]$. The DAFNE experiments on $\phi \rightarrow K^{0}$ $+\bar{K}^{0}$ are particularly interesting in this regard, because of the two-particle correlation at production. However, as just mentioned, we need more understanding of the possible impact of nonlinear effects in this case.

The suggestion $[6,30]$ that neutrinos might be sensitive to dissipative quantum-gravitational effects is very interesting. ${ }^{8}$ As discussed above, we do not believe that the Lindblad formalism is necessarily applicable, at least in the form discussed so far in the literature [6,8]. A different issue is that of Lorentz invariance. The formalism of $[1,2]$ is not Lorentz invariant, and we have suggested an approximate treatment for ultra-relativistic particles such as photons [31] or neutrinos [32], which we believe to be the most appropriate starting point for an analysis of neutrino data [5]. In this approach, the velocity of a photon (or massless) neutrino deviates from $c$, which is identified as the low-energy limit of the velocity of light:

$$
v=c \times\left[1-\frac{E}{M}+\cdots\right]
$$

where $E$ is the energy and $M$ is some large mass scale that might be $\mathcal{O}\left(M_{P}\right)$. This deviation from $c$ has the characteristics of a refractive index in vacuo. In addition, there may be stochastic fluctuations in the velocity of a photon (or neutrino) of specified energy that have a diffusive character. These suggestions arise from our considerations of recoil effects on quantum-gravitational vacuum fluctuations due to the passage of an energetic particle [5]. Timing observations of distant astrophysical sources are sensitive to $M \sim 10^{15}$ $\mathrm{GeV}$, and there are prospects to increase this to $M \sim M_{P}$ or beyond [31].

\footnotetext{
${ }^{8}$ There is also a suggestion to probe these via double- $\beta$ decay experiments [30].
} 
If only the refractive index effect (43) is present and the quantum-gravitational mass parameter $M$ is flavor independent, there would be no practical consequences for neutrino oscillation physics. However, consequences would ensue if $M$ is flavor dependent or if there are also diffusive effects. We plan to return to these issues in a future publication.

\section{ACKNOWLEDGMENTS}

We thank Eligio Lisi for arousing our interest in this question. The work of N.E.M. is partially supported by the P.P.A.R.C. (U.K.). The work of D.V.N. is partially supported by DOE grant DE-F-G03-95-ER-40917. N.E.M. and D.V.N. also thank H. Hofer for his interest and support.
[1] J. Ellis, J. Hagelin, D. Nanopoulos, and M. Srednicki, Nucl. Phys. B241, 381 (1984).

[2] J. Ellis, N.E. Mavromatos and D.V. Nanopoulos, From Supersymmetry to the Origin of Space Time, Erice Subnuclear Series Vol. 31 (World Scientific, Singapore, 1993), p. 1; Mod. Phys. Lett. A 10, 1685 (1995); for an updated review, see J. Ellis, N. E. Mavromatos, and D. V. Nanopoulos, Chaos, Solitons Fractals 10, 345 (1999).

[3] J. Ellis, N.E. Mavromatos, and D.V. Nanopoulos, Phys. Lett. B 293, 142 (1992); Int. J. Mod. Phys. A 11, 1489 (1996); J. Ellis, J.L. Lopez, N.E. Mavromatos, and D.V. Nanopoulos, Phys. Rev. D 53, 3846 (1996); CPLEAR Collaboration, R. Adler et al., J. Ellis, J.L. Lopez, N.E. Mavromatos, and D.V. Nanopoulos, Phys. Lett. B 364, 239 (1995).

[4] P. Huet and M. Peskin, Nucl. Phys. B434, 3 (1995).

[5] For a recent review of this and other medium effects, see J. Ellis, N.E. Mavromatos and D.V. Nanopoulos, invited contribution to the 2nd International Conference on "Physics Beyond the Standard Model: Beyond the Desert 99: Accelerator, Nonaccelerator and Space Approaches,', Ringberg Castle, Tegernsee, Germany, 1999, gr-qc/9909085.

[6] E. Lisi, A. Marrone, and D. Montanino, Phys. Rev. Lett. 85, 1166 (2000).

[7] G. Lindblad, Commun. Math. Phys. 48, 119 (1976); for a review, see V. Gorini et al., Rep. Math. Phys. 13, 149 (1978).

[8] S. Adler, Phys. Rev. D 62, 117901 (2000) and references therein.

[9] M. Czachor and M. Kuna, Phys. Rev. A 58, 128 (1998).

[10] F. Benatti and R. Floreanini, Nucl. Phys. B488, 335 (1997); B511, 550 (1998); Ann. Phys. (N.Y.) 273, 58 (1999); Phys. Lett. B 468, 287 (1999).

[11] J. Ellis, N.E. Mavromatos, and D.V. Nanopoulos, Int. J. Mod. Phys. A 12, 2639 (1997); 13, 1059 (1998); for a recent review see Proceedings of the 4th International Symposium on Sources and Detection of Dark Matter in the Universe (DM 2000), Marina del Rey, California, 2000 (Springer, Berlin, in press), gr-qc/0005100.

[12] N. Gisin and I.C. Percival, J. Phys. A 26, 2233 (1993); N. Gisin, Helv. Phys. Acta 62, 363 (1989).

[13] W.H. Zurek, Phys. Today 44(10), 36 (1991); see also A. Albrecht, Phys. Rev. D 46, 5504 (1992).

[14] G. Shore, Nucl. Phys. B286, 349 (1987); H. Osborn, Phys. Lett. B 222, 97 (1989); Ann. Phys. (N.Y.) 200, 1 (1990), and references therein.

[15] F. David, Mod. Phys. Lett. A 3, 1651 (1988); J. Distler and H. Kawai, Nucl. Phys. B321, 509 (1989).

[16] I. Antoniadis, C. Bachas, J. Ellis, and D.V. Nanopoulos, Phys.
Lett. B 211, 393 (1988); Nucl. Phys. B328, 117 (1989); Phys. Lett. B 257, 278 (1991).

[17] S. Coleman, Nucl. Phys. B310, 643 (1998).

[18] A.B. Zamolodchikov, Pis'ma Zh. Éksp. Teor. Fiz. 43, 565 (1986) [JETP Lett. 43, 730 (1986)]; Yad. Fiz. 46, 1819 (1987) [Sov. J. Nucl. Phys. 46, 1090 (1987)].

[19] E. Witten, Phys. Rev. D 44, 314 (1991).

[20] J. Polchinski, Phys. Rev. Lett. 75, 4724 (1995); in Fields, Strings and Duality, Proceedings of TASI 96, edited by C. Efthimiou and B.R. Greene (World Scientific, Singapore, 1997), p. 293.

[21] I.I. Kogan, N.E. Mavromatos, and J.F. Wheater, Phys. Lett. B 387, 483 (1996).

[22] N.E. Mavromatos and R.J. Szabo, Phys. Rev. D 59, 104018 (1999).

[23] V. Gurarie, Nucl. Phys. B410, 535 (1993); J.S. Caux, I.I. Kogan, and A.M. Tsvelik, ibid. B466, 444 (1996); M.A.I. Flohr, Int. J. Mod. Phys. A 11, 4147 (1996); M.R. Gaberdiel and H.G. Kausch, Nucl. Phys. B489, 293 (1996); I.I. Kogan and N.E. Mavromatos, Phys. Lett. B 375, 111 (1996); M.R. Rahimi-Tabar, A. Aghamohammadi, and M. Khorrami, Nucl. Phys. B497, 555 (1997); I.I. Kogan, A. Lewis, and O.A. Soloviev, Int. J. Mod. Phys. A 13, 1345 (1998).

[24] N.E. Mavromatos and R.J. Szabo, Phys. Lett. B 430, 94 (1998).

[25] N.E. Mavromatos and D.V. Nanopoulos, Int. J. Mod. Phys. B 11, 851 (1997).

[26] M. Goulian and M. Li, Phys. Rev. Lett. 66, 2051 (1991).

[27] I. Kogan, Phys. Lett. B 265, 269 (1991).

[28] J. Schwinger, J. Math. Phys. 2, 407 (1961); for recent reviews, see E. Calzetta and B.L. Hu, Phys. Rev. D 37, 2878 (1988); E. Calzetta, S. Habib, and B.L. Hu, ibid. 37, 2901 (1988); H. Umezawa, Advanced Field Theory: Micro, macro and thermal concepts (AIP, New York, 1993).

[29] N.E. Mavromatos and R.J. Szabo, Int. J. Mod. Phys. A (to be published), hep-th/9909129.

[30] H.V. Klapdor-Kleingrothaus, H. Pas, and U. Sarkar, Eur. Phys. J. A 8, 577 (2000).

[31] G. Amelino-Camelia, J. Ellis, N.E. Mavromatos, and D.V. Nanopoulos, Int. J. Mod. Phys. A 12, 607 (1997); G. AmelinoCamelia, J. Ellis, N.E. Mavromatos, D.V. Nanopoulos, and S. Sarkar, Nature (London) 393, 763 (1998); J. Ellis, K. Farakos, N.E. Mavromatos, V.A. Mitsou, and D.V. Nanopoulos, Astrophys. J. 535, 139 (2000).

[32] J. Alfaro, H.A. Morales-Tecotl, and L.F. Urrutia, Phys. Rev. Lett. 84, 2318 (2000); J. Ellis, N.E. Mavromatos, D.V. Nanopoulos, and G. Volkov, Gen. Relativ. Gravit. 32, 1777 (2000). 